\title{
ELF Radar System Proposed for Localized D-Region Ionospheric Anomalies
}

\author{
Jamesina J. Simpson, Student Member, IEEE, and Allen Taflove, Fellow, IEEE
}

\begin{abstract}
This letter proposes a novel extremely low frequency (ELF) radar for localized D-region (altitude $<95 \mathrm{~km}$ ) ionospheric anomalies that have been generated by natural geophysical processes. The proposed system would use the former U.S. Navy Wisconsin Transmitting Facility as a distant well-characterized impulsive ELF source. Sample calculations that demonstrate how passive vertical $E$-field detectors could characterize ionospheric conductivity depressions of variable diameter located above Los Angeles are provided. These calculations have been obtained using our recently developed three-dimensional whole-Earth electromagnetic wave propagation model based upon the rigorous finite-difference time-domain solution of Maxwell's equations. A key potential application of the proposed ELF radar system is the detection of hypothesized ionospheric earthquake precursors.
\end{abstract}

Index Terms-Earthquakes, extremely low frequency (ELF), finite difference time domain (FDTD), ionospheric disturbances, radar, remote sensing.

\section{INTRODUCTION}

D ESPITE substantial ground- and satellite-based technology for studying the upper ionosphere, extracting the electron densities versus height in the D-region $(<95 \mathrm{~km})$ is still a difficult problem, particularly at night [1]. As a result, monitoring and understanding anomalous behavior of the D-region due to geophysical, solar, or other phenomena is not a well-developed field of science. To date, techniques for studying the D-region have included in situ measurements by rockets [2], or studying the spectrum from uncontrolled (random and naturally occurring) sources, such as intense lightning discharges [1]. These methods, however, have limited range, do not offer a practical means of continuously monitoring the lower ionosphere, and are difficult or impossible to implement in many regions of the world.

A few examples of probing the D-region using controlled (man-made) radio-frequency sources exist. For example, continuous-wave very low frequency (VLF: $3-30 \mathrm{kHz}$ ) to low frequency (LF: $30-300 \mathrm{kHz}$ ) signals propagating in the Earthionosphere waveguide from communication and navigation systems have been analyzed to infer if any ionospheric anomalies exist between the source and the receiver [3], [4].

In this letter, we propose a novel extremely low frequency (ELF: $3-300 \mathrm{~Hz}$ ) radar for locating and characterizing localized ionospheric anomalies that have been formed within $\sim 100 \mathrm{~km}$ of the Earth's surface by natural geophysical processes. Our

Manuscript received March 17, 2006; revised April 19, 2006. This work was supported by the Pittsburgh Supercomputing Center under Grant EAR050012P.

The authors are with the Department of Electrical and Computer Engineering, Northwestern University, Evanston, IL 60208 USA (e-mail: j-simpson@northwestern.edu).

Digital Object Identifier 10.1109/LGRS.2006.878443 system assumes: 1) operation of the former U.S. Navy Wisconsin Transmitting Facility (WTF) as a distant well-characterized impulsive ELF source to illuminate the ionospheric anomaly and 2) passive detection of the resulting vertical electric $(E)$ field time-waveform at the Earth's surface in the vicinity of the ionospheric anomaly. (Note that the ELF illumination is assumed to be of sufficiently low power to have negligible heating or any other effects upon the ionospheric anomaly.) Employing a man-made impulsive ELF source such as the WTF could allow, in principle, a continuous and systematic monitoring scheme that avoids variabilities arising from naturally occurring sources such as lightning, which have random properties.

To illustrate the operation of our proposed system, we provide three calculations of vertical $E$-field signals at the Earth's surface in Los Angeles (which is selected arbitrarily) below bowl-shaped ionospheric conductivity depressions. The depressions are assumed to have a depth of $20 \mathrm{~km}$ and a radius of either 100, 200, or $380 \mathrm{~km}$. Remote excitation is provided by a $76-\mathrm{Hz}$ electromagnetic-wave pulse launched essentially isotropically from the WTF. Our calculations utilize a recently developed three-dimensional (3-D) geodesic-grid wholeEarth electromagnetic wave propagation model [5] based upon the rigorous finite-difference time-domain (FDTD) solution of Maxwell's equations [6]. This model provides a direct 3-D time-domain calculation of round-the-world ELF propagation accounting for arbitrary horizontal as well as vertical geometrical and electrical inhomogeneities/anisotropies of the excitation, ionosphere, lithosphere, and oceans. Furthermore, the geodesic grid-cell arrangement is more efficient and provides more isotropic wave propagation than our previous 3-D latitude-longitude-grid FDTD models of the global Earthionosphere waveguide [7]-[10]. The results of our modeling work show that a simple measurement of the vertical $E$-field signal below a localized ionospheric depression could provide its location, size, shape, and depth.

\section{Three-Dimensional Geodesic FDTD Grid}

We will now briefly describe the arrangement of the 3-D FDTD grid used in this study. For a more detailed explanation, the reader is referred to [5]. Fig. 1 illustrates the distribution of cells as seen from a constant radial coordinate for a sample geodesic grid (courtesy of [11]), which, for our purpose, corresponds to the transverse-magnetic (TM) case of Maxwell's equations. The grid of Fig. 1 shows a total of 642 planar cells, of which 630 are hexagons and 12 are pentagons. Interestingly, there are exactly 12 pentagons regardless of the grid resolution. For efficient mapping into the computer memory, this grid can be divided into five equal panels of size $i m 5 \times j m$ cells, 


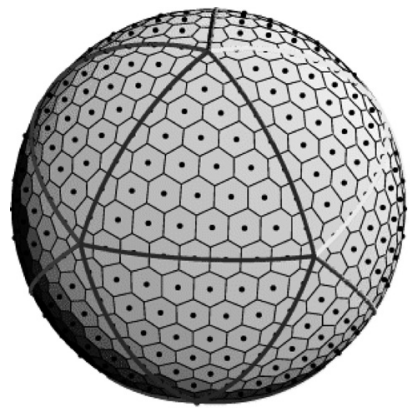

(a)

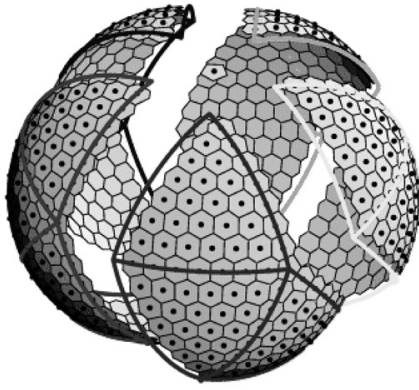

(b)
Fig. 1. General layout of a 642-cell geodesic grid covering the complete Earth-sphere as seen from a constant radial coordinate (courtesy of [11]). The grid can be divided into five equal sections. As described in [11], each grid cell can easily be assigned logically Cartesian coordinates.

where $i m 5=18$ and $j m=10$, as described in [11]. These five grid panels can then easily be assigned logically Cartesian coordinates [11]. This powerful interpretation of a spherical geodesic grid for purposes of efficient computer processing can be implemented for a variety of meshing densities over the sphere [11].

A fully 3-D FDTD grid requires alternating planes of TM and transverse-electric (TE) field components. For our purpose, the complementary TE planes are comprised entirely of triangular cells centered at the vertices of each hexagonal and pentagonal cell of the TM planes [5].

Brewer et al. [12] describe an optimization procedure for selecting the areas and locations of the TM hexagonal and pentagonal grid cells (and thereby also the TE triangular cells) on the sphere to ensure numerical consistency and to maximize the order of accuracy in the context of the Laplace operator. We have found that this optimization procedure also maximizes the observed accuracy of wave propagation about the sphere as governed by Maxwell's equations [5].

\section{Details of the FDTD ELF RADAR Study}

In this letter, our goal is to determine how the presence of a naturally occurring ionospheric depression influences the radial (vertical) $E$-field component $\left(E_{\mathrm{r}}\right)$ at the Earth's surface below the depression when illuminated by an ELF pulse radiated from a distant transmitter. (Note that the ELF illumination is assumed to be of sufficiently low power to have negligible heating or any other effects upon the ionospheric anomaly.) Specifically, we model three cases of ionospheric conductivity anomalies located above Los Angeles at $118^{\circ} \mathrm{W}, 34^{\circ} \mathrm{N}$. The anomalies considered are assumed to be bowl-shaped ionospheric conductivity depressions having a depth of $20 \mathrm{~km}$ and a radius of either 100,200 , or $380 \mathrm{~km}$.

As the ELF source, we assume the WTF section of the Navy site located near Clam Lake, WI $\left(90.9^{\circ} \mathrm{W}, 46.5^{\circ} \mathrm{N}\right)$. This facility was in operation until September 2004 [13] and is located at a distance of $\sim 2.8 \mathrm{Mm}$ from the ionospheric depression above Los Angeles. We assume nominal operation of the WTF with two orthogonal ground lines, each $22.5 \mathrm{~km}$ long and carrying a current of $300 \mathrm{~A}$, one oriented in the north-south direction and the other in the east-west direction. The transmitted signal is a pulse generated by the double-sideband amplitude modulation of a $76-\mathrm{Hz}$ carrier by a $43-\mathrm{ms}$ Gaussian envelope waveform (full-width at half-maximum). Below the WTF, we model the Laurentian Plateau (or Canadian Shield), which is a large region of low-conductivity rock of 2.4E-4 S/m that extends northward from the Great Lakes toward the Arctic Ocean and includes much of Canada and Greenland.

We use two separate FDTD models to calculate the time waveforms of the surface $E_{\mathrm{r}}$ component. Model A serves to provide the reference time waveforms (i.e., no ionospheric anomaly present). Model B provides the time waveforms for the case of the ionospheric anomaly present.

Both Models A and B utilize topographic and bathymetric data from the NOAA-NGDC "Global Relief CD-ROM." These data are mapped onto each 3-D space lattice having 163842 total cells at each radial coordinate, yielding an assumed resolution of about $63 \mathrm{~km}$ between adjacent hexagon/pentagon cell centers. In the radial direction, there are 40 grid cells, yielding a resolution of $5 \mathrm{~km}$ between a depth of $100 \mathrm{~km}$ into the lithosphere and an altitude of $100 \mathrm{~km}$. These assumed resolutions yield 3-D FDTD grids, each having a total of over 6.5 million cells.

For all grid points in the lithosphere of Models A and B that are not part of the Laurentian Plateau below the WTF, conductivity values are assigned according to [14]. Conductivity values are selected depending upon the location of an $E$ component (i.e., below an ocean or within a continent). For grid points in the atmosphere of Models A and B, daytime and nighttime exponential conductivity profiles used in [15] are assumed, with dawn occurring at $0^{\circ}$ longitude. The effective waveguide height of reflection for these profiles is approximately $48 \mathrm{~km}$ for day and $76 \mathrm{~km}$ for night [15] for the unperturbed conditions. At the center of the ionospheric depression occurring at nighttime above Los Angeles, the effective waveguide height of reflection is about $56 \mathrm{~km}$.

\section{Computational Modeling Results}

We define the percent increase in the peak vertical $E$-field power $\Delta P$ due to the ionospheric depression as the absolute value of the difference between the square of the peak surface $E_{\mathrm{r}}$ value of Models $\mathrm{A}$ and B divided by the square of the peak surface $E_{\mathrm{r}}$ value of the Model A waveform. Fig. 2 is a map of the calculated $\Delta P$ for the 100 -km-radius $20-\mathrm{km}$-deep ionospheric depression above Los Angeles at $118^{\circ} \mathrm{W}, 34^{\circ} \mathrm{N}$. In Fig. 2, we observe an $\sim 4.5 \%$ maximum perturbation of the incident signal due to the ionospheric depression. Fig. 2 also illustrates that both the size and shape of the ionospheric anomaly can be determined using an array of passive $E$-field detectors at the Earth's surface.

Fig. 3 is a map of the calculated $\Delta P$ for the $200-\mathrm{km}$-radius 20-km-deep ionospheric depression above Los Angeles. We see that there is an $\sim 7.5 \%$ maximum perturbation of the incident signal due to this anomaly. Similarly, Fig. 4 is a map of $\Delta P$ for the 380-km-radius 20-km-deep ionospheric anomaly above Los Angeles. Here, we see that there is an $\sim 8 \%$ maximum perturbation of the incident signal due to this anomaly. As for the case of Fig. 2, the ionospheric depressions of Figs. 3 and 4 can be characterized using passive $E$-field detectors for spatial extent. Further, at any location, the downward extent of 


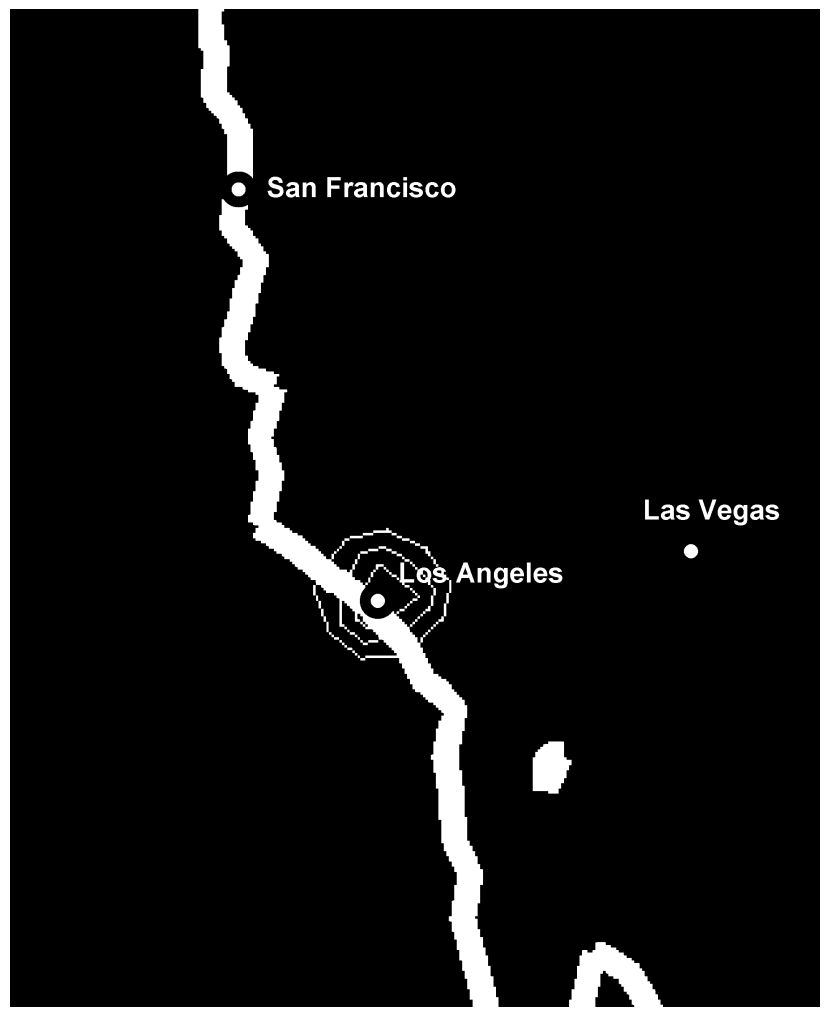

Fig. 2. Map of the percent increase in the peak vertical $E$-field power $\Delta P$ for the bowl-shaped 100-km-radius 20-km-deep ionospheric depression above Los Angeles at $118^{\circ} \mathrm{W}, 34^{\circ} \mathrm{N}$. Each contour line represents a $1 \%$ increase in $\Delta P$, yielding a value of over $4 \%$ at the center.

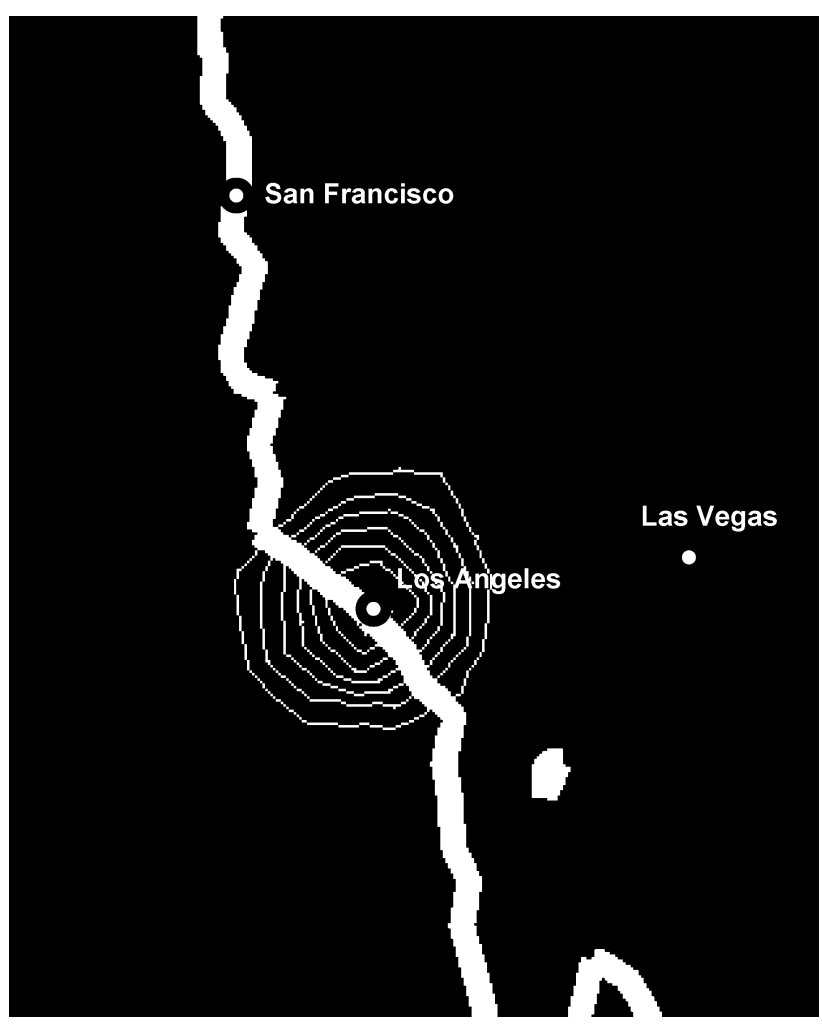

Fig. 3. Map of the percent increase in the peak vertical $E$-field power $\Delta P$ for the bowl-shaped 200-km-radius 20-km-deep ionospheric depression above Los Angeles at $118^{\circ} \mathrm{W}, 34^{\circ} \mathrm{N}$. Each contour line represents a $1 \%$ increase in $\Delta P$, yielding a value of over $7 \%$ at the center.

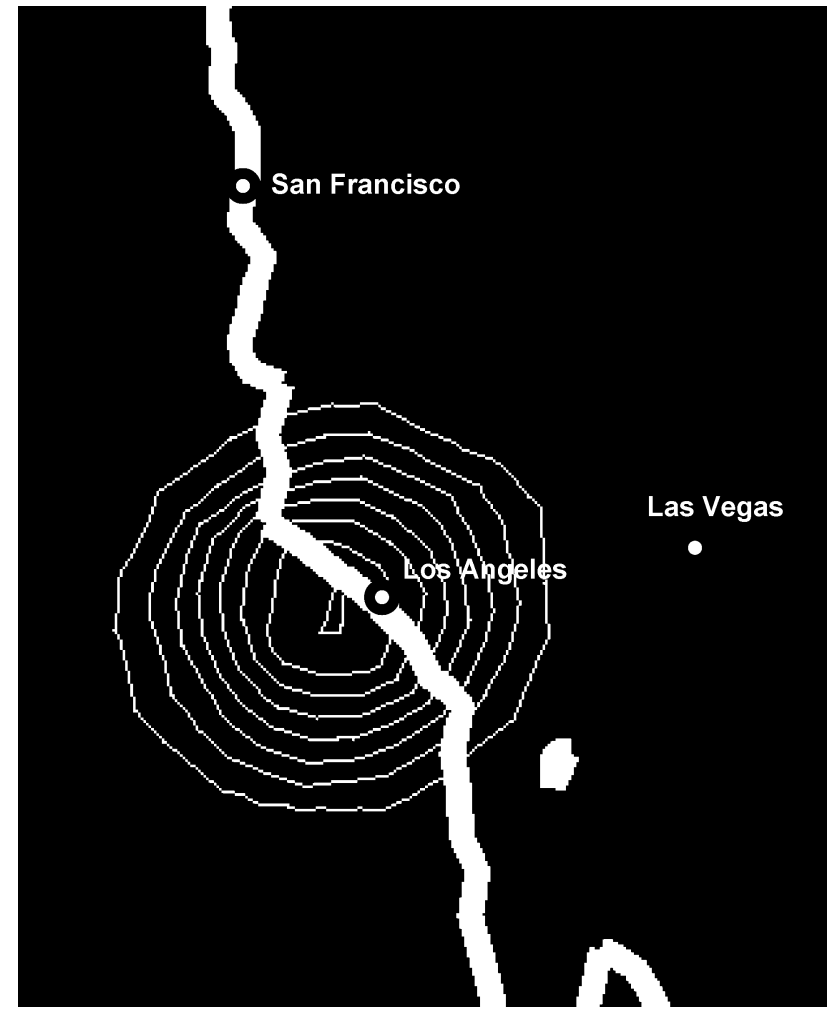

Fig. 4. Map of the percent increase in the peak vertical $E$-field power $\Delta P$ for the bowl-shaped 380-km-radius 20-km-deep ionospheric depression above Los Angeles at $118^{\circ} \mathrm{W}, 34^{\circ} \mathrm{N}$. Each contour line represents a $1 \%$ increase in $\Delta P$, yielding a value of over $8 \%$ at the center.

the depression from the regular ionospheric conditions can be extracted from the perturbation level occurring at that point.

Finally, Fig. 5 illustrates the calculated surface $E_{\mathrm{r}}$ time waveform at Los Angeles at $118^{\circ} \mathrm{W}, 34^{\circ} \mathrm{N}$ for the case of: 1) no ionospheric depression present; 2) a 100-km-radius 20-km-deep ionospheric depression above Los Angeles; 3) a 200-km-radius 20-km-deep ionospheric depression above Los Angeles; and 4) a 380-km-radius 20-km-deep ionospheric depression above Los Angeles. Fig. 5(a) shows the first $40 \mathrm{~ms}$ as the pulse propagates through Los Angeles after radiating from the WTF. Fig. 5(b) shows an enlarged version of the four cases of Fig. 5(a) between 22 and $30 \mathrm{~ms}$. We observe that for ionospheric depressions of larger radii, the delay of the slow-tail response increases monotonically. This is yet a second indication of the existence and radius of the ionospheric depression, which can be used in conjunction with measurement of $\Delta P$ to strengthen the observation.

\section{CONCLUSION AND ONGOING WORK}

In this letter, we have proposed a novel ELF radar for D-region diagnostics to sense ionospheric depressions within $\sim 100 \mathrm{~km}$ of the Earth's surface. To illustrate this new technology, we provided numerical simulation results of a $76-\mathrm{Hz}$ pulse emitted from the former U.S. Navy site in Wisconsin for passive sounding of $20-\mathrm{km}$ ionospheric depressions that have been generated by natural geophysical processes locally above Los Angeles. For this study, we employed our 3-D geodesic FDTD model of the entire Earth-ionosphere cavity to map out 


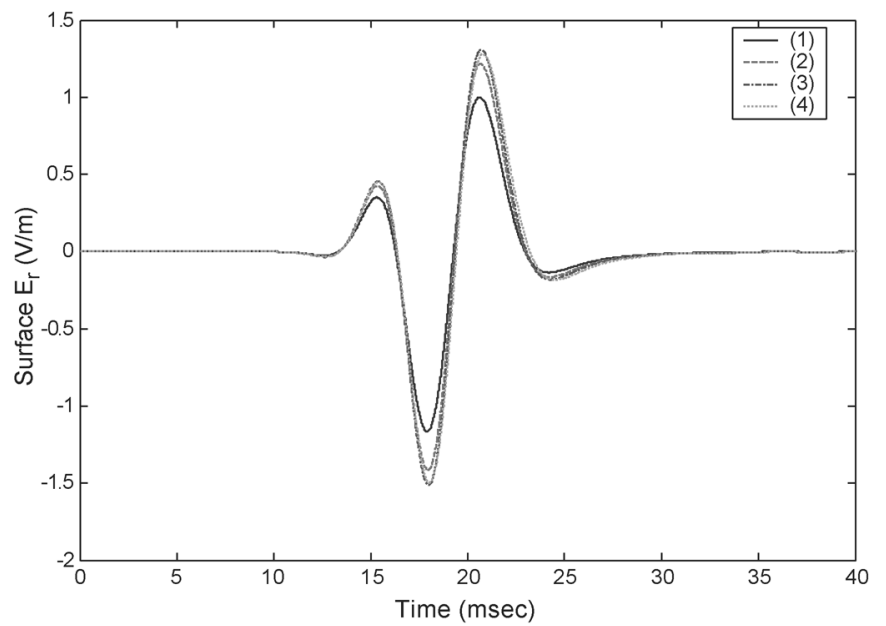

(a)

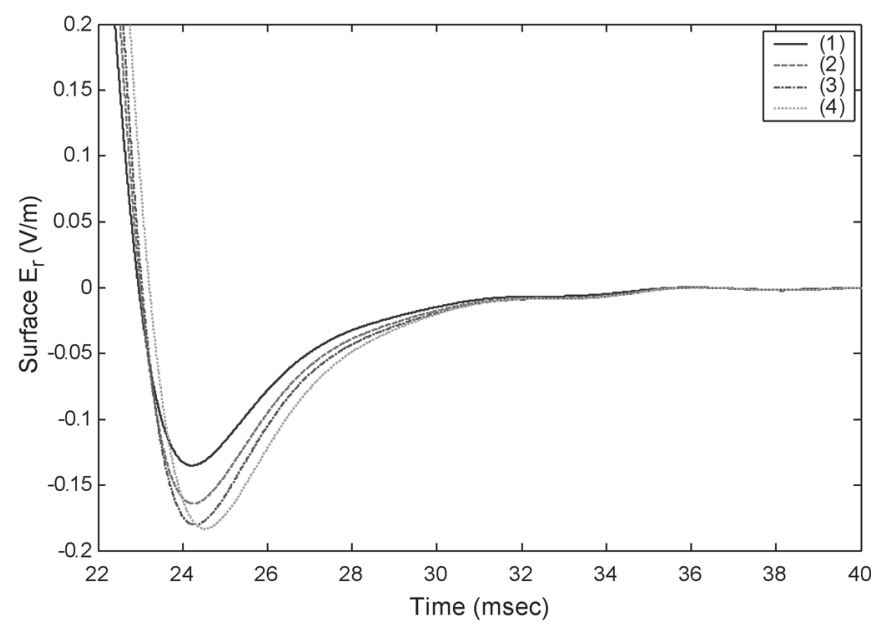

(b)

Fig. 5. (a) Surface $E_{\mathrm{r}}$ time waveform at Los Angeles at $118^{\circ} \mathrm{W}, 34^{\circ} \mathrm{N}$ for the case of: 1) no ionospheric depression present; 2) a 100-km-radius $20-\mathrm{km}$ deep ionospheric depression above Los Angeles; 3) a 200-km-radius 20-kmdeep ionospheric depression above Los Angeles; and 4) a 380-km-radius 20-km-deep ionospheric depression above Los Angeles. (b) Enlargement of the surface $E_{\mathrm{r}}$ time-waveform slow-tail response at Los Angeles for the four cases shown in (a). We observe that for ionospheric depressions of larger radii, the delay of the slow-tail response increases monotonically.

the percent increase in the vertical $E$-field power at the Earth's surface. Our results indicate that passive $E$-field detectors at the Earth's surface can be used to determine the location, size, shape, and depth of ionospheric depressions within $\sim 100 \mathrm{~km}$ of the Earth's surface.

Several practical applications exist for our proposed ELF system. For example, it has been proposed that the electron density at altitudes of the nighttime ionospheric D-region increases considerably above earthquake preparation zones (see, for example, [16] and [17]). This increase in electron density, which is hypothesized to exist prior to earthquakes having magnitude $M \geq 5$, can be equated to a lowering of the effective height of the ionosphere, with the radius of the anomaly dependent upon the magnitude of the impending earthquake [16]. However, although there is much literature on this subject, there is no current understanding of potential interactions between earthquake preparation processes and the lower ionosphere. Our
ELF radar for ionospheric disturbances could therefore provide useful information regarding localized ionospheric depressions hypothesized to occur as earthquake precursors. Additional applications include remote sensing of ionospheric conditions related to other geophysical processes as well as radio propagation. Further, this application involving the WTF complements (could be used in conjunction with) our previously proposed ELF radar for major oil deposits [5], [9].

Our ongoing work in this area includes modeling/designing a potential global ELF radar for ionospheric anomalies. We are also continuing our investigation of ultra-low-frequency (ULF: 0-3 Hz) and ELF precursors to earthquakes. Finally, we are developing FDTD models of the Earth-ionosphere waveguide that include plasma phenomena in the upper atmosphere.

\section{ACKNOWLEDGMENT}

The authors would like thank R. Heikes (Colorado State University) for his technical assistance with the geodesic grids.

\section{REFERENCES}

[1] S. A. Cummer, U. S. Inan, and T. F. Bell, "Ionospheric D region remote sensing using VLF radio atmospherics," Radio Sci., vol. 33, no. 6, pp. 1781-1792, 1998.

[2] C. F. Sechrist, Jr., "Comparisons of techniques for measurement of D-region electron densities," Radio Sci., vol. 9, no. 2, p. 137, 1974.

[3] O. A. Molchanov, M. Hayakawa, T. Ondah, and E. Kawai, "Precursory effects in the subionospheric VLF signals for the Kobe earthquake," Phys. Earth Planet. Inter., vol. 105, no. 3-4, pp. 239-248, 1998.

[4] C. J. Rodger, M. A. Clilverd, and N. R. Thomson, "Modeling of subionospheric VLF signal perturbations associated with earthquakes," Radio Sci., vol. 34, no. 5, pp. 1177-1185, 1999.

[5] J. J. Simpson, R. P. Heikes, and A. Taflove, "FDTD modeling of a novel ELF radar for major oil deposits using a three-dimensional geodesic grid of the Earth-ionosphere waveguide," IEEE Trans. Antennas Propag., vol. 54, no. 6, pp. 1734-1741, Jun. 2006.

[6] A. Taflove and S. C. Hagness, Computational Electrodynamics: The Finite-Difference Time-Domain Method, 3rd ed. Norwood, MA: Artech House, 2005

[7] J. J. Simpson and A. Taflove, "Three-dimensional FDTD modeling of impulsive ELF propagation about the Earth-sphere," IEEE Trans. Antennas Propag., vol. 52, no. 2, pp. 443-451, Feb. 2004.

[8] _ "Electrokinetic effect of the Loma Prieta Earthquake calculated by an entire-Earth FDTD solution of Maxwell's equations," Geophys. Res. Lett., vol. 32, no. 9, p. L09302, 2005.

[9] — , "A novel ELF radar for major oil deposits," IEEE Geosci. Remote Sens. Lett., vol. 3, no. 1, pp. 36-39, Jan. 2006.

[10] A. Taflove and J. J. Simpson, "Introduction to Maxwell's equations and the Yee algorithm," in Computational Electrodynamics: The Finite-Difference Time-Domain Method, 3rd ed. A. Taflove and S. C. Hagness, Eds. Norwood, MA: Artech House, 2005, pp. 85-103.

[11] D. A. Randall, T. D. Ringler, and R. P. Heikes, "Climate modeling with spherical geodesic grids," Comput. Sci. Eng., vol. 4, no. 5, pp. 32-41, Sep. 2002.

[12] M. Brewer, L. Freitag-Diachin, P. Knupp, T. Leurent, and D. Melander, "The mesquite mesh quality improvement toolkit," in Proc. 12th Int. Meshing Roundtable, Santa Fe, NM, 2003, pp. 239-250.

[13] J. Garza. (2004, Sep. 17). Navy pulls plug on embattled ELF site. Milwaukie Journal Sentinel. [Online]. Available: www.jsonline.com

[14] J. Hermance, "Electrical conductivity of the crust and mantle," in Global Earth Physics: A Handbook of Physical Constants. Washington, DC: AGU, 1995.

[15] P. Bannister, "The determination of representative ionospheric conductivity parameters for ELF propagation in the Earth-ionosphere waveguide," Radio Sci., vol. 20, no. 4, pp. 977-984, 1985.

[16] S. Pulinets and K. Boyarchuk, Ionospheric Precursors of Earthquakes. Berlin, Germany: Springer-Verlag, 2004

[17] V. P. Kim, S. A. Pulinets, and V. V. Hegai, "Theoretical model of possible disturbances in the nighttime mid-latitude ionospheric D region over an area of strong-earthquake preparation," Radiophys. Quantum Electron., vol. 45 , no. 4 , pp. 262-268, 2002. 